\title{
Toward transcriptional therapies for the failing heart: chemical screens to modulate genes
}

\author{
Timothy A. McKinsey' and Eric N. Olson²
}

${ }^{1}$ Myogen Inc., Westminster, Colorado, USA. ${ }^{2}$ Department of Molecular Biology, University of Texas Southwestern Medical Center, Dallas, Texas, USA.

\begin{abstract}
In response to acute and chronic stresses, the heart frequently undergoes a remodeling process that is accompanied by myocyte hypertrophy, impaired contractility, and pump failure, often culminating in sudden death. The existence of redundant signaling pathways that trigger heart failure poses challenges for therapeutic intervention. Cardiac remodeling is associated with the activation of a pathological gene program that weakens cardiac performance. Thus, targeting the disease process at the level of gene expression represents a potentially powerful therapeutic approach. In this review, we describe strategies for normalizing gene expression in the failing heart with small molecules that control signal transduction pathways directed at transcription factors and associated chromatin-modifying enzymes.
\end{abstract}

\section{Introduction}

Heart failure, a complex disorder in which cardiac contractility is insufficient to meet the metabolic demands of the body, is the leading cause of death in the Western world. Approximately 5 million individuals in the United States $(2-3 \%$ of the population) are afflicted with this syndrome, and the numbers are rising. Heart failure results from diverse acute and chronic insults, including coronary artery disease, myocardial infarction, hypertension, valve abnormalities, and inherited mutations in sarcomere and cytoskeletal proteins.

Currently, heart transplantation represents the most effective therapy for end-stage heart failure, but this approach obviously cannot reach the millions of affected individuals worldwide and is not suitable for patients with milder forms of the disease. Traditional therapies for heart failure have involved the use of multiple drugs to improve cardiac contractile function by modifying neurohumoral signaling (e.g., $\beta$ blockers and angiotensin-converting enzyme inhibitors) or normalizing calcium handling by the cardiomyocyte (1). While such strategies promote short-term improvement in cardiac function, the 5-year mortality rate for heart failure patients remains close to $50 \%$. Thus, there is a great need for the development of novel therapeutics, preferably new drugs, that will improve the quality of life and prolong survival of heart failure patients. An understanding of the mechanistic underpinnings of heart failure represents an essential step toward that goal.

Heart failure is frequently preceded by pathological enlargement of the heart due to hypertrophy of cardiac myocytes (2-5). Cardiac hypertrophy and failure are accompanied by the reprogramming of cardiac gene expression and the activation of "fetal" cardiac genes, which encode proteins involved in contraction, calcium handling, and metabolism (Figure 1) (6-9). Such transcriptional reprogramming has been shown to correlate with loss of cardiac function

Nonstandard abbreviations used: CaMK, calcium/calmodulin-dependent protein kinase; HAT, histone acetyltransferase; HDAC, histone deacetylase; 5-HT, 5-hydroxytryptamine; MCIP, modulatory calcineurin-interacting protein; MEF2, myocyte enhancer factor-2: MHC, myosin heavy chain; NAD, nicotinamide adenine dinucleotide; NFAT, nuclear factor of activated T cells; PAMH, pyridine activator of myocyte hypertrophy.

Conflict of interest: The authors own stock in Myogen Inc.

Citation for this article: J. Clin. Invest. 115:538-546 (2005).

doi:10.1172/JCI200524144 and, conversely, improvement in cardiac function in response to drug therapy or implantation of a left ventricular assist device is accompanied by normalization of cardiac gene expression (10-12). Strategies to control cardiac gene expression, therefore, represent attractive, albeit challenging, approaches for heart failure therapy.

Pharmacological normalization of cardiac gene expression in the settings of hypertrophy and heart failure will require the identification of new drug targets that serve as nodal regulators to integrate and transmit stress signals to the genome of the cardiac myocyte. Transcription factors are generally considered to be poor drug targets due to their lack of enzymatic activity and inaccessibility in the nucleus. However, we and others have recently found that cardiac stress response pathways control cardiac gene expression by modulating the activities of chromatin-remodeling enzymes, which act as global regulators of the cardiac genome during pathological remodeling of the heart (13). Here we describe strategies for manipulating chromatin structure to alter cardiac gene expression in the settings of pathological hypertrophy and heart failure as a new means of "transcriptional therapy" for these disorders. We focus on pathways and mechanisms that govern the activity of the nuclear factor of activated T cells (NFAT) and myocyte enhancer factor-2 (MEF2) transcription factors, which integrate cardiac stress signals and play pivotal roles in transcriptional reprogramming of the hypertrophic and failing heart.

\section{Transcriptional remodeling of the hypertrophic and failing heart}

In response to acute and chronic insults, the adult heart undergoes distinct remodeling responses, which can take the form of ventricular wall thickening, accompanied by myocyte hypertrophy; or dilatation, accompanied by myocyte elongation (eccentric hypertrophy), serial assembly of sarcomeres, and myocyte apoptosis. While there may be salutary aspects of cardiac hypertrophy, for example, the normalization of ventricular wall stress, it is clear that prolonged hypertrophy in response to stress is deleterious and is a major predictor for heart failure and sudden death $(2-5)$. On the other hand, physiological hypertrophy, as occurs in highly trained athletes or during normal postnatal development, represents a beneficial form of cardiac growth. A major challenge in designing potential therapies for cardiac hypertrophy and failure is to selectively target components of pathological signaling 


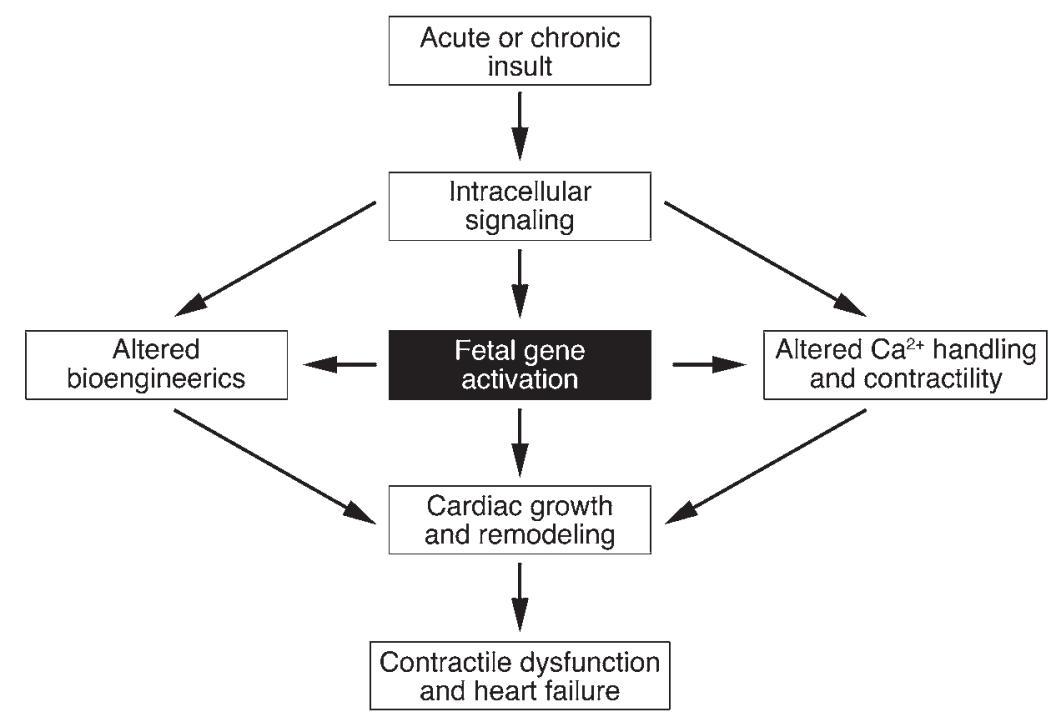

Figure 1

Abnormalities associated with cardiac remodeling during pathological hypertrophy and heart failure.

mechanisms without affecting mechanisms of physiological cardiac growth and function.

Heart failure is typically a disorder of pump function, although it can also arise from acute volume overload (acute aortic insufficiency), high-output disorders (thyroid hormone excess), and pericardial restriction. A hallmark of maladaptive cardiac growth and remodeling is the differential regulation of the 2 myosin heavy chain (MHC) isoforms, $\alpha$ and $\beta$, which has a profound effect on cardiac function (14). $\alpha$-MHC, which is upregulated in the heart after birth, has high ATPase activity, whereas $\beta$-MHC has low ATPase activity. Pathological remodeling of the heart in rodent models is accompanied by upregulation of $\beta$-MHC expression and downregulation of $\alpha$-MHC, with consequent reduction in myofibrillar ATPase activity and reduced shortening velocity of cardiac myofibers, leading to eventual contractile dysfunction. Remarkably, minor changes in $\alpha-\mathrm{MHC}$ content of the heart can have a profound influence on cardiac performance (15).

Whereas $\beta$-MHC is the predominant $\mathrm{MHC}$ isoform in the adult rodent heart, the adult human heart contains primarily $\alpha-M H C$, which has led some to doubt the relevance of $\mathrm{MHC}$ isoform switching in human heart failure. Nonetheless, there is compelling evidence indicating a role for changes in $\mathrm{MHC}$ isoform expression in the pathogenesis of heart failure in humans. Indeed, $\alpha-\mathrm{MHC}$ mRNA and protein levels are markedly reduced in failing human hearts $(16,17)$, and improvement of left-ventricular ejection fraction through $\beta$ blocker therapy is associated with normalization of $\alpha$-MHC expression (10). Additionally, a mutation in the human $\alpha-M H C$ gene was identified in association with hypertrophic cardiomyopathy (18), which demonstrates that, despite its low abundance, $\alpha-\mathrm{MHC}$ is critical for normal heart function and further validates the hypothesis that MHC isoform switching plays a significant role in human heart failure.

In addition to the $\alpha-\mathrm{MHC} / \beta-\mathrm{MHC}$ switch, the gene encoding the sarcoplasmic reticulum $\mathrm{Ca}^{2+}$ ATPase (SERCA) is commonly downregulated in the hypertrophic and failing heart, with consequent loss of efficient calcium cycling. Conversely, genes encoding the natriuretic peptides atrial natriuretic factor (ANF) and B-type natriuretic peptide (BNP) are upregulated as a compensatory mechanism to promote natriuresis and suppress myocyte hypertrophy (14). During pathological remodeling, the heart also undergoes a shift in its mode of energy utilization from oxidative toward glycolytic, which may further contribute to cardiac demise (19). Modulation of RNA polymerase II activity by cyclin-dependent protein kinases has been shown to contribute to many of the abovementioned processes in the diseased heart (20).

\section{Signaling pathways leading to cardiac hypertrophy and failure}

Diverse neurohumoral signals acting through numerous, interwoven signal transduction pathways lead to pathological cardiac hypertrophy and heart failure (6-9). Many such agonists act through cell surface receptors coupled with Gaq to mobilize intracellular calcium, with consequent activation of downstream kinases and the calcium- and calmodulin-dependent phosphatase calcineurin. MAPK signaling pathways are also interconnected at multiple levels with calcium-dependent kinases and calcineurin (21). The details of these pathways have been reviewed elsewhere $(6-9,22)$. $\beta$-Adrenergic agonists also influence cardiac growth and function through the generation of cAMP, which activates PKA and other downstream effectors (23). While we focus in this review on the roles of transcriptional regulators as mediators of cardiac stress-response pathways, it is important to emphasize that such signaling pathways also target a variety of substrates in the cardiomyocyte, including components of the contractile apparatus, calcium channels, and their regulatory proteins.

\section{Control of gene expression by histone acetylation/deacetylation}

In eukaryotes, histone-dependent packaging of genomic DNA into chromatin is a central mechanism for gene regulation. The basic unit of chromatin, the nucleosome, comprises DNA wrapped around a histone octamer. Nucleosomes interact to create a highly compact structure that limits access of transcriptional machinery to genomic DNA, thereby repressing gene expression (24).

Histone acetyltransferases (HATs) and histone deacetylases (HDACs) act in an opposing manner to control the acetylation state of nucleosomal histones (Figure 2A). Acetylation of the conserved amino-terminal histone tails by HATs is thought to relax nucleosomal structure by weakening the interaction of the positively charged histone tails with the negatively charged phosphate backbone of DNA, allowing access of transcriptional activators and gene induction. Deacetylation of nucleosomal histones by HDACs results in transcriptional repression.

There are multiple mammalian HDACs, which fall into 3 classes on the basis of structural and biochemical characteristics (25). Class I HDACs consist primarily of a catalytic domain, while class II HDACs contain amino-terminal extensions of approximately 500 residues that harbor binding sites for other transcriptional regulators. The amino-terminal extensions of class II HDACs also contain conserved phosphorylation sites that serve as targets for signaling pathways, thereby connecting signals from outside the cell to the genome. Class III HDACs are 
A

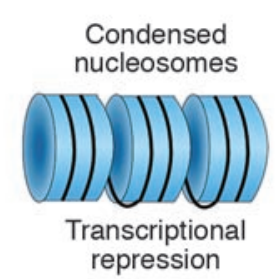

B

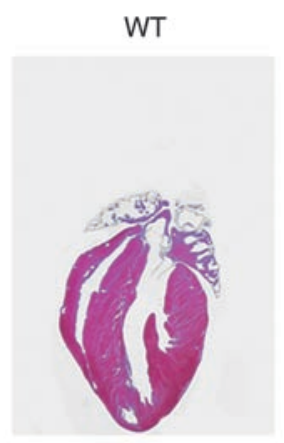

Relaxed nucleosomes

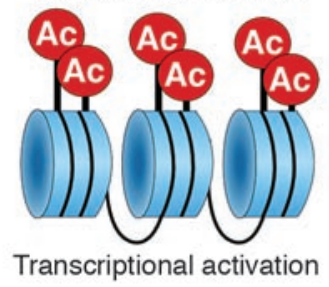

HDAC9 KO $\times$ HDAC9-KO

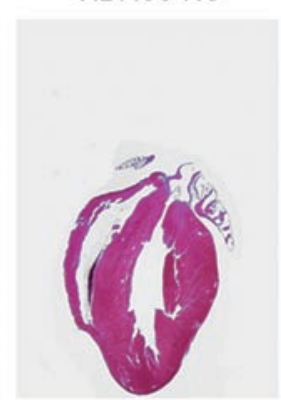

Calcineurin- $\mathrm{Tg}$

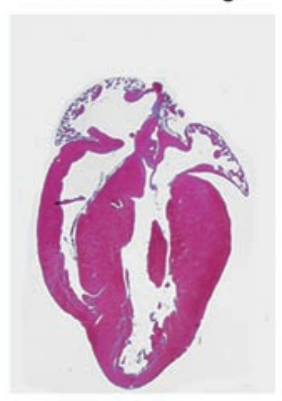

Calcineurin-Tg

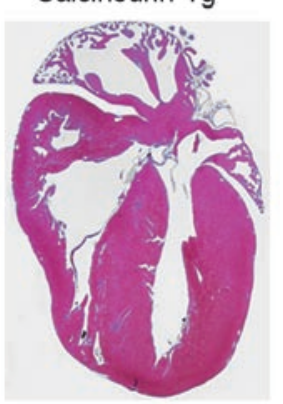

Figure 2

Model of HDAC function and hypersensitivity of HDAC9-knockout mice to cardiac stress.

(A) Schematic of chromatin structure and the actions of HATs and HDACs. HATs acetylate (Ac) histones, causing relaxation of nucleosomal structure and transcriptional activation. HDACs oppose the actions of HATs by deacetylating histones, causing chromatin condensation and transcriptional repression. (B) Histological sections of adult mouse hearts of the indicated genotypes are shown. HDAC9-null mice do not display a cardiac phenotype at early age. The cardiac calcineurin transgene (Calcineurin- $\mathrm{Tg}$ ) induces dramatic cardiac growth, which is exacerbated in the absence of HDAC9. Adapted with permission from Cell (26). unique in that they require nicotinamide adenine dinucleotide (NAD) for catalytic activity.

\section{Control of cardiac growth by class II HDACs}

Evidence supporting a role for HDACs in the control of stressinduced cardiac remodeling initially came from studies of class II HDACs. Forced overexpression of class II HDACs 5 or 9 in cardiac myocytes prevents hypertrophy in response to diverse agonists (26-28). More important, mice in which the gene encoding either HDAC5 or HDAC9 has been disrupted by homologous recombination are hypersensitive to pathological signals, developing cardiomegaly and eventual cardiac failure in response to stresses such as pressure overload or introduction of the calcineurin transgene (Figure 2B) (26-29).

Abnormal cardiac growth in HDAC-knockout animals correlates with superactivation of the MEF2 transcription factor (26), which suggests a causal relationship between MEF2 activity and the development of cardiac pathology. Indeed, prior studies established that MEF2 factors selectively associate with class II HDACs via an 18-amino-acid motif present only in these HDACs. Class II HDACs form a complex with MEF2 on gene regulatory elements, resulting in repression of genes harboring MEF2 binding sites (30).

\section{Posttranslational regulation of class II HDACs}

Class II HDAC levels do not appear to change in stressed myocardium $(26,29)$. Instead, these HDACs are shuttled from the nucleus to the cytoplasm in response to stress (Figure 3), which provides a posttranslational mechanism to override HDACmediated repression of cardiac growth $(27,28,31)$. This redistribution of HDACs frees MEF2 (and other transcription factors) to associate with HATs $(32,33)$, resulting in increased local histone acetylation and activation of downstream genes that promote cell growth.

Nucleocytoplasmic shuttling of class II HDACs is dependent on phosphorylation of 2 serine-containing motifs found exclusively in these HDACs (34-36). When phosphorylated, these motifs associate with a chaperone protein, termed 14-3-3, which results in the unmasking of a class II HDAC nuclear export sequence (NES) (35-38). The NES is subsequently bound by the CRM1 nuclear export receptor, which escorts class II HDACs from the nucleus to the cytoplasm (Figure 4).

\section{Figure 3}

Agonist-dependent nuclear export of HDAC5 correlates with cardiomyocyte hypertrophy. Stimulation of cardiomyocytes with neurohumoral agonists evokes a hypertrophic response characterized by fetal gene activation, sarcomere assembly, and hypertrophy. The upper panels show the subcellular distribution of HDAC5 fused to GFP. In unstressed myocytes, HDAC5-GFP is localized to the nucleus, whereas stimulation with a hypertrophic agonist causes it to redistribute to the cytoplasm. A small molecular inhibitor of the signaling pathway from the cell surface to HDAC5 prevents nuclear export of HDACs and blocks hypertrophy, as detected by staining for sarcomeric $\alpha$-actinin.
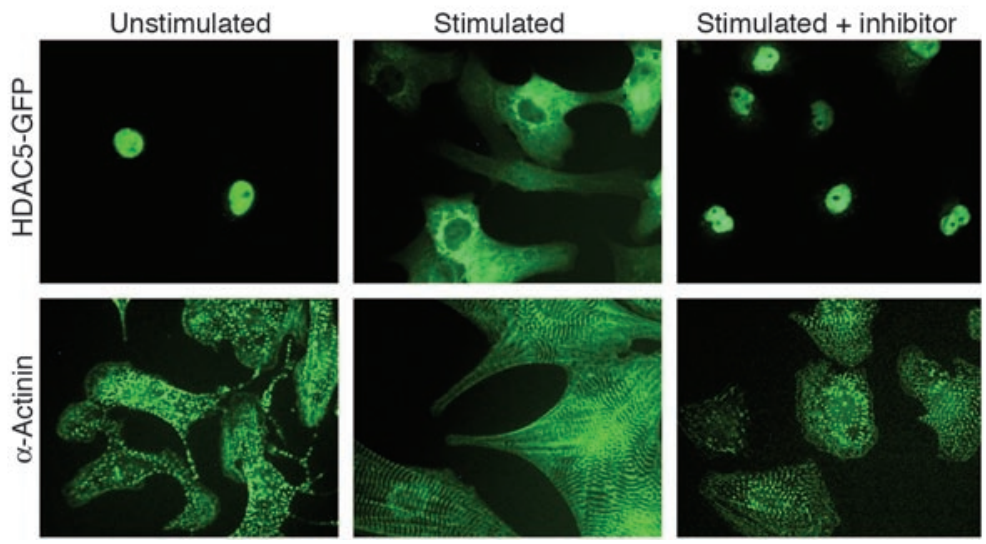


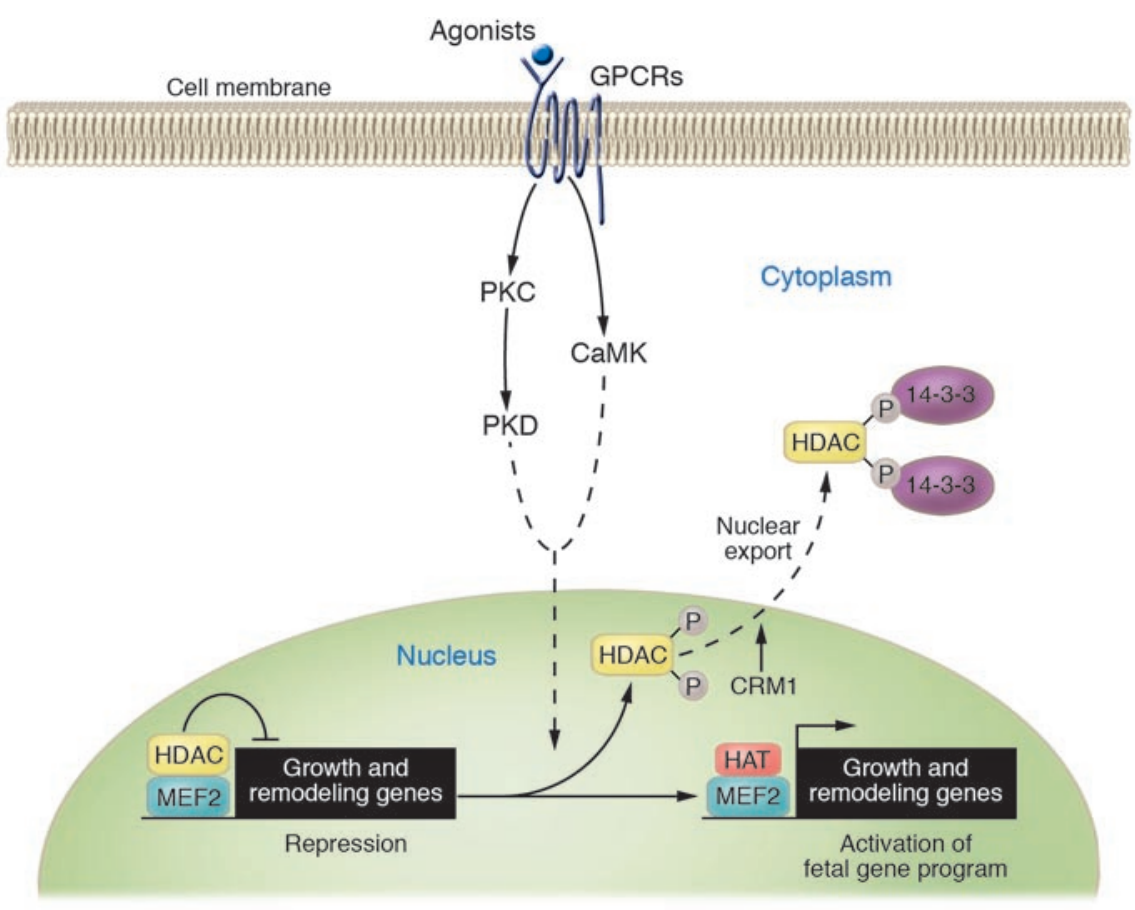

\section{Figure 4}

Signal-dependent modulation of cardiac genes and hypertrophy by class II HDACs. MEF2 recruits class II HDACs to target genes, which results in transcriptional repression due to chromatin condensation. Stimulation of cardiomyocytes with neurohumoral agonists acting through G-protein coupled receptors (GPCRs) activates kinase pathways that culminate with the phosphorylation of class II HDACs and their export to the cytoplasm as a complex with 14-3-3 proteins. The nuclear export protein CRM1 is required for HDAC nuclear export. The release of class II HDACs from MEF2 allows for the association of HATs with MEF2 and consequentially chromatin relaxation and transcriptional activation of fetal cardiac genes.
Signal-dependent neutralization of class II HDACs is a key step in the control of stress-mediated cardiac growth. Thus, there is interest in identifying signaling molecules that govern posttranslational modification and trafficking of these transcriptional regulators. The availability of small molecules that specifically sustain the repressive function of class II HDACs by inhibiting such signaling factors should provide a novel means to control pathological cardiac remodeling. Initial studies demonstrated that calcium/calmodulin-dependent protein kinase (CaMK) is a potent class II HDAC kinase (34). However, an important issue was whether CaMK is the sole kinase responsible for regulating HDAC nuclear export in the heart or whether multiple kinases might converge on the regulatory HDAC phosphorylation sites, and thus different HDAC kinases might be activated in response to different stimuli.

We recently showed that signaling via PKC leads to the phosphorylation of the same sites in HDAC5 that are phosphorylated by CaMK (28). The PKC family includes at least 12 different isoforms, many, but not all, of which are expressed at appreciable levels in the myocardium (39). Direct activation of PKC by phorbol ester is sufficient to induce nuclear export of HDAC5, and hypertrophic agonists stimulate nuclear export of HDAC5 in cardiac myocytes through a signaling pathway that depends on PKC activation (28).

PKCs are unable to directly phosphorylate HDACs and instead modulate HDAC phosphorylation via the downstream effector kinase PKD. PKD, which is phosphorylated and activated by PKC, physically associates with HDAC5 and promotes phosphorylation of the 14-3-3 binding sites on the protein, which results in HDAC nuclear export. Importantly, small molecule inhibitors that target PKC and PKD, but not CaMK, abolish agonist-mediated nuclear export of HDAC5 cardiac myocytes, which suggests a predominant role for this pathway in the control of HDAC5 in the heart.

\section{PKD inhibitors}

Numerous kinase inhibitors are in development for a variety of clinical indications (40). Our data suggest that PKD inhibitors could be used to block cardiac remodeling in settings where class II HDACs are subject to signal-dependent neutralization. To date, PKD-specific inhibitors have not been identified. However, a heterocyclic derivative of the general serine/threonine kinase inhibitor staurosporine, termed Gö-6976, has been shown to function as a potent $\mathrm{PKD}$ inhibitor (41). Although Gö-6976 blocks the activity of several kinases in addition to PKD, it may be possible to synthesize analogs with enhanced specificity.

PKD was recently shown to have the capacity to phosphorylate cardiac troponin I (cTnI), resulting in enhanced contractility (42). Whether PKD is a bona fide TnI kinase, and the implications of this finding on the potential for PKD inhibitors as heart failure therapeutics awaits further investigation. It should also be noted that PKD is expressed in many cell types in addition to cardiomyocytes, and has been implicated in processes as diverse as Golgi transport, proliferation, and apoptosis (43). Clearly, PKD inhibitors may have effects, both positive and negative, in the heart and in other tissues. In vivo proof-of-concept testing with PKD inhibitors awaits the identification of novel bioavailable small molecules with potent and selective activity against this kinase. High-throughput screening and medicinal chemistry approaches may reveal such compounds.

A potential complication of therapeutically targeting a single kinase such as PKD to control class II HDACs in the heart is the possible existence of redundant kinases able to bypass the blockade of PKD. An approach to circumvent this problem may involve inhibiting events that occur following HDAC phosphorylation, such as dissociation of phospho-HDAC from MEF2 or HDAC shuttling to the cytosol.

\section{MEF2/HDAC — additional considerations}

It is important to emphasize that HDACs and HDAC kinases do not function in isolation to control MEF2 activity. Indeed, MEF2 is regulated by a plethora of signaling networks that include MAPKs, as well as the calcineurin phosphatase $(30,44)$. One mechanism 


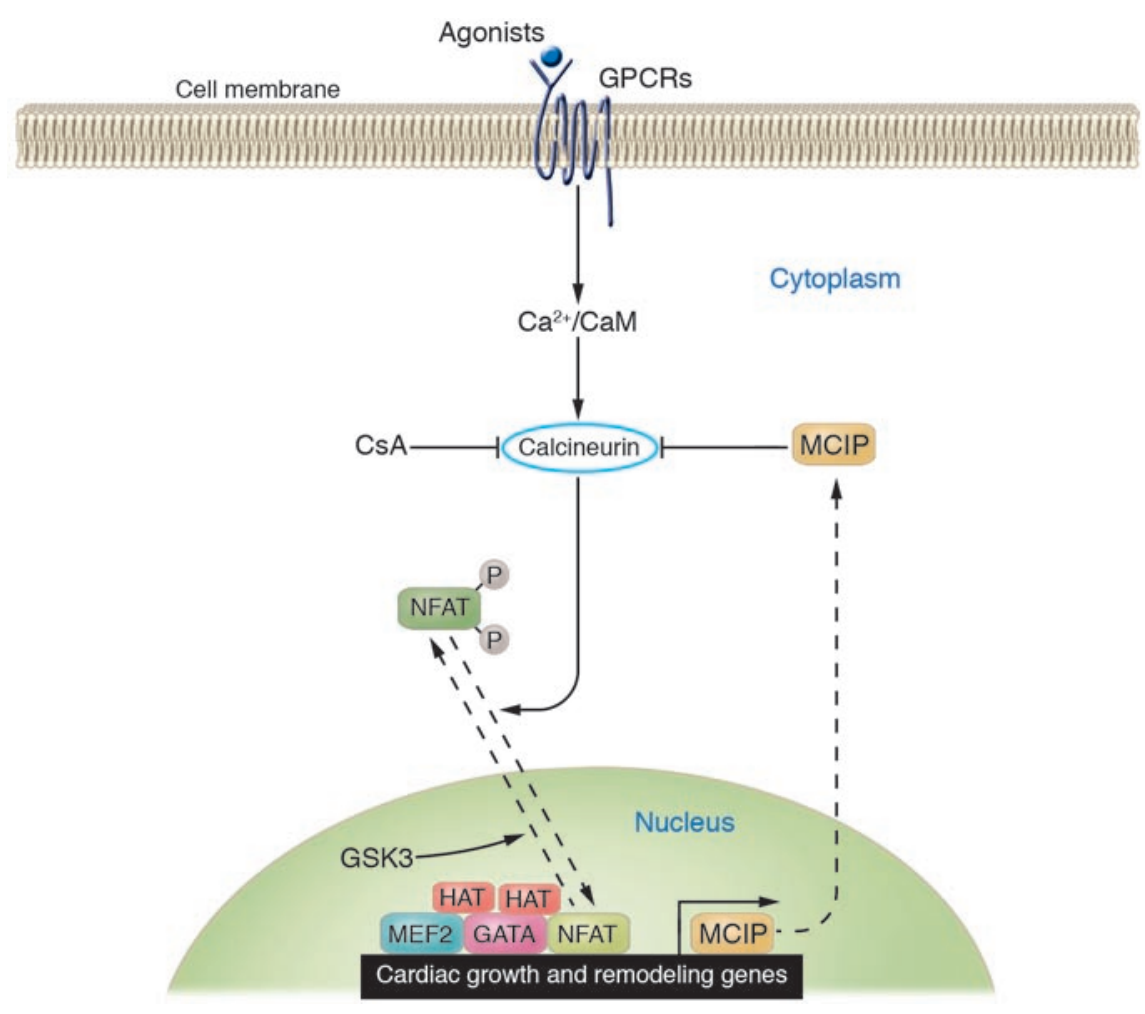

\section{Figure 5}

Signal-dependent modulation of cardiac genes and hypertrophy by calcineurin/NFAT signaling. Stimulation of cardiomyocytes with neurohumoral agonists acting through GPCRs activates calcineurin, which dephosphorylates NFAT, allowing its entry into the nucleus, where it acts with other transcription factors, such as GATA4, to activate stress-response genes. Glycogen synthase kinase-3 (GSK3) phosphorylates the serine residues dephosphorylated by calcineurin, driving NFAT back to the cytoplasm and terminating the growth signal. The MCIP1 gene is a target of calcineurin/NFAT signaling, and its protein product associates with calcineurin to restrain its activity, thereby creating a negative feedback loop to govern cardiac growth.

(50). The ability of this single activated transcription factor to substitute for upstream stress signals underscores the importance of transcriptional control in the pathogenesis of cardiac hypertrophy and heart failure. The prohypertrophic action of calcineurin can be suppressed by the activity of glycogen synthase kinase-3 (GSK3) (52-54), which phosphorylates the calcineurin sub-

for calcineurin-dependent activation of MEF2 involves NFAT transcription factors, which, upon dephosphorylation by calcineurin, translocate to the nucleus and facilitate recruitment of HATs to MEF2-response elements (see below) (45).

A challenge in developing inhibitors of MEF2 (or other components of signaling pathways) will be to ensure that sufficient MEF2 activity is maintained for homeostatic control in the heart. Indeed, ablation of MEF2 in the heart can lead to dilated cardiomyopathy in mice and has been linked to increased risk of myocardial infarction in humans $(46,47)$. These phenotypes may be related to the ability of MEF2 to regulate expression of the PPAR $\gamma$ coactivator- $1 \alpha$ (PGC-1) transcription factor, a master regulator of mitochondrial biogenesis (48). In what may be a related finding, cardiomyopathy induced by cyclin-dependent kinase 9 signaling was recently shown to be associated with repression of MEF2-mediated PGC-1 expression (49).

\section{Control of cardiac growth by the calcineurin/NFAT axis}

Kinase cascades can certainly regulate cardiac gene expression through negative effects on HDACs. However, protein phosphatases likely play an equally important role in the regulation of chromatin structure during cardiac remodeling. In this regard, the calcium- and calmodulin-dependent protein phosphatase calcineurin is activated in response to cardiac stress signaling, and its activation has been shown to be sufficient and, in many cases, necessary for pathological cardiac hypertrophy $(50,51)$. Calcineurin dephosphorylates members of the NFAT family of transcription factors, which enables them to translocate into the nucleus, where they activate transcription in cooperation with other transcription factors, including MEF2 and GATA4 (Figure 5).

Misexpression of a mutant form of NFAT, which lacks the regulatory phosphorylation sites and therefore mimics the activated form of the protein, is sufficient to induce cardiac hypertrophy in vivo strate sites in NFAT proteins, thereby driving NFAT from the nucleus to the cytoplasm and preventing hypertrophy.

NFAT factors activate gene expression, in part, by recruiting HATs to gene regulatory elements harboring NFAT and MEF2 binding sites. Thus, calcineurin inhibitors should block the ability of NFAT to trigger chromatin-remodeling events that stimulate expression of genes required for pathological cardiac growth. Consistent with this hypothesis, inhibition of calcineurin signaling with cyclosporin A or FK-506 can prevent cardiac hypertrophy in animal models $(50,51$, $55)$. However, since these compounds also suppress $T$ cell function and have other side effects, they will not likely be suitable for longterm treatment of patients with heart failure.

There has been interest in developing small molecules that specifically suppress calcineurin/NFAT signaling in the heart and thereby circumvent the undesirable immunosuppressive effects associated with global calcineurin inhibition. In this regard, calcineurin signaling is inhibited by a family of muscle-enriched proteins, referred to as modulatory calcineurin-interacting proteins (MCIPs) (56), which associate with the calcineurin catalytic subunit and restrain its activity. Thus, it may be possible to selectively suppress calcineurin in the heart by enhancing the expression of MCIP or its association with calcineurin.

MCIP1 expression is activated by calcineurin through a series of NFAT binding sites in the promoter of the MCIP1 gene, which provides a calcineurin-dependent negative feedback loop whereby the heart can restrain potentially deleterious calcineurin signaling (Figure 5) (57). Consistent with the notion that MCIP1 acts as an endogenous suppressor of calcineurin, MCIP1-knockout mice are hypersensitive to calcineurin signaling and rapidly develop fatal cardiac hypertrophy in response to calcineurin activation (Figure 6) (58). Conversely, overexpression of MCIP in the heart protects against diverse stress signals and blocks hypertrophy (Figure 6) 


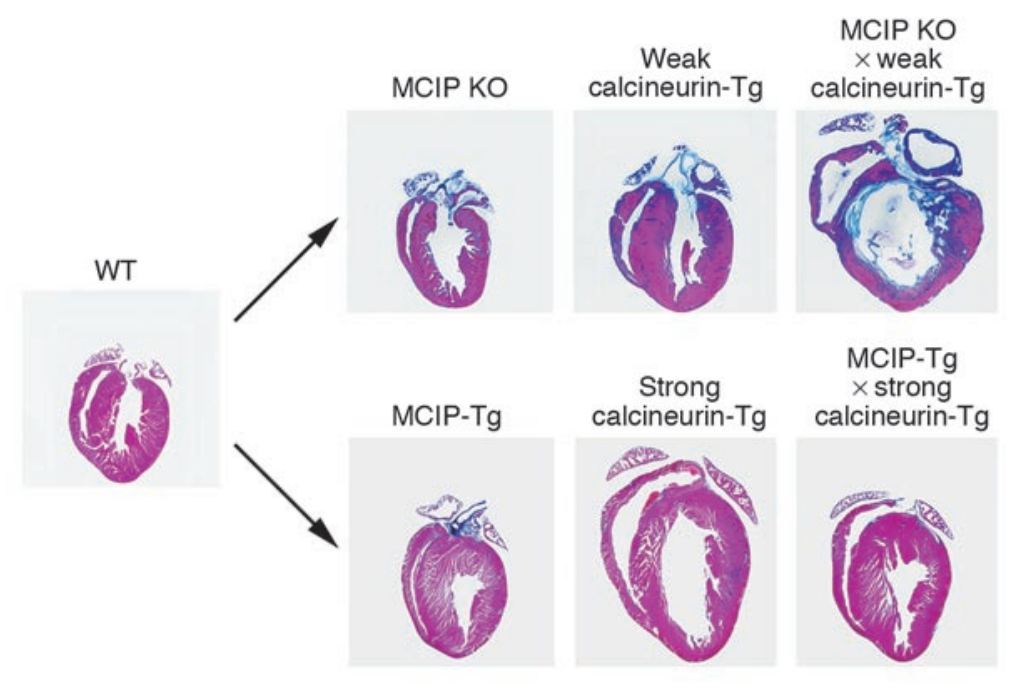

\begin{abstract}
Figure 6
Modulation of cardiac calcineurin signaling by MCIP expression. The upper set of panels shows the effect of calcineurin signaling in MCIP1-knockout mice. In the absence of MCIP, the heart is sensitized to calcineurin signaling and rapidly undergoes fatal hypertrophy. Because of the heightened sensitivity of MCIP1-knockout mice to calcineurin, only a weak calcineurin transgene can be expressed in the absence of MCIP1. A stronger calcineurin transgene, as used in the lower panels, results in heart failure and death within the first few days after birth. The lower set of panels shows the effect of calcineurin signaling in mice that overexpress MCIP1 in the heart. Overexpression of MCIP1 prevents pathological hypertrophy. Adapted with permission from Proceedings of the National Academy of Sciences of the United States of America $(58,59)$.
\end{abstract}

$(59,60)$. MCIP overexpression has also been shown to sustain cardiac function and enhance survival following myocardial infarction in mice (61). Importantly, blockade to hypertrophy by MCIP overexpression does not result in cardiac decompensation, which supports the notion that strategies to prevent pathological hypertrophy will not necessarily be counterproductive $(55,59,60)$. MCIP also plays a permissive role in calcineurin activation, the basis of which is incompletely understood (58). However, the inhibitory activity of MCIP toward calcineurin is dominant under conditions of MCIP overexpression.

\section{A chemical screen for modulators of the calcineurin/NFAT axis}

Based on the apparent cardioprotective functions of MCIP1, a high-throughput screen for small molecular activators of MCIP1 expression in cardiac myocytes was designed (27). In principle, such activators could induce MCIP1 expression through stimulation of the calcineurin/NFAT signaling pathway, in which case they would likely be prohypertrophic, or they could act through other mechanisms. In a study using regulatory DNA sequences from the MCIP1 gene linked to a luciferase reporter, several novel small molecules capable of inducing luciferase expression were identified (27). One such activator, pyridine activator of myocyte hypertrophy (PAMH), dramatically stimulated MCIP1 expression.

Scrutiny of the structure of PAMH revealed an embedded pharmacophore resembling serotonin (5-hydroxytryptamine [5-HT]) (Figure 7). Indeed, 5-HT receptor antagonists abolished PAMH activity on cardiomyocytes, and direct ligand binding assays showed that $\mathrm{PAMH}$ binds the $5-\mathrm{HT}_{2 \mathrm{~B}}$ receptor with an affinity of approximately $60 \mathrm{nM}(27)$.

Through analysis of the effects of a series of small molecule inhibitors on PAMH activity, PAMH was found to induce MCIP1 expression by activating calcineurin with consequent nuclear import of NFAT and by promoting the phosphorylation-dependent nuclear export of class II HDACs. Thus, although PAMH potently upregulates MCIP expression, it does so through mechanisms that are predicted to exacerbate pathological remodeling of the heart. Indeed, PAMH was found to dramatically stimulate hypertrophy of cultured myocytes. Given this shortcoming, additional screens were devised to identify small molecules that elevate cardiac MCIP protein levels through calcineurin-independent mechanisms.
Although PAMH is unlikely to progress as a development candidate for heart failure, it has altered the paradigm for cardiac calcineurin signaling and has uncovered novel possibilities for manipulation of calcium signaling in the heart. Because PAMH is able to mimic 5-HT, we screened structurally related compounds to find one that might also modulate calcineurin signaling via the $5-\mathrm{HT}_{2 \mathrm{~B}}$ receptor and discovered another novel 4-amino pyridine, A-PAMH, which antagonizes the activity of PAMH (27). Intriguingly, A-PAMH also blocks the hypertrophic effects of PE, which acts through the $\alpha$-adrenergic receptor. We do not currently understand the molecular basis for this antagonism, which could reflect crosstalk between the $5-\mathrm{HT}_{2 \mathrm{~A} / \mathrm{B}}$ and $\alpha$-adrenergic receptors, competition between A-PAMH and PE for binding to the $\alpha$-adrenergic receptor, or another mechanism.

Consistent with the finding that signaling through $5-\mathrm{HT}_{2 \mathrm{~A} / \mathrm{B}}$ receptors can drive cardiac growth, overexpression of the $5-\mathrm{HT}_{2 \mathrm{~B}}$ receptor in the heart leads to cardiac hypertrophy (62), and $5-\mathrm{HT}_{2 \mathrm{~B}}$ receptor-knockout mice die during mid-gestation from a failure of ventricular growth (63). We propose a model in which $5-\mathrm{HT}_{2 \mathrm{~A} / \mathrm{B}}$ signaling promotes cardiac hypertrophy by stimulating calcineurin/NFAT signaling, with consequent recruitment of HATs to regulatory regions of NFAT target genes. Since PAMH also stimulates nuclear export of class II HDACs, it is likely that MEF2 plays a role in the mechanism by which $5-\mathrm{HT}_{2 \mathrm{~A} / \mathrm{B}}$ signaling triggers cardiac remodeling.

\section{Normalization of cardiac gene expression with HDAC inhibitors}

In addition to indirectly regulating chromatin structure via small molecules that target signaling pathways, it may also be possible

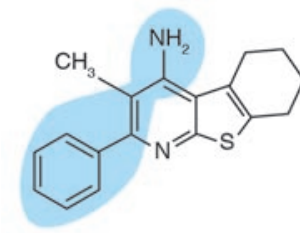

$\mathrm{PAMH}$

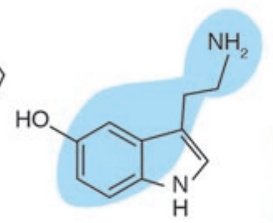

Serotonin

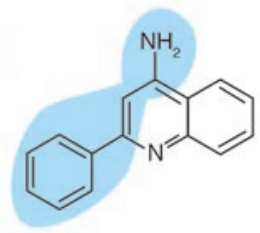

A-PAMH
Figure 7

Structures of serotonin, PAMH, and A-PAMH. 

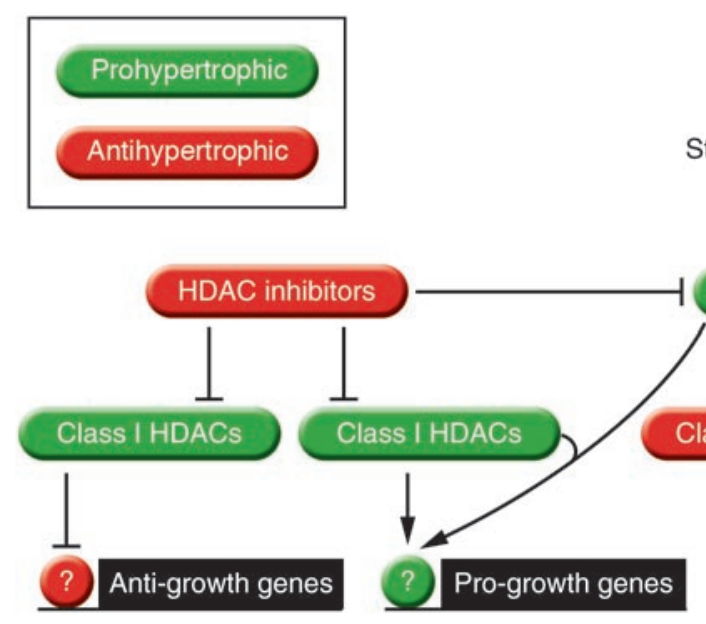
Kinases

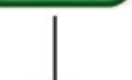

Class II HDACs

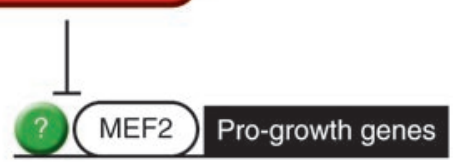

\section{Figure 8}

Potential roles of HDACs in cardiac myocytes. Class II HDACs repress hypertrophy. Their activity is repressed by signal-dependent phosphorylation. Class I HDACs appear to be prohypertrophic based on the ability of HDAC inhibitors to prevent hypertrophy. Class I HDACs could act by inhibiting prohypertrophic signaling pathways, by inhibiting the expression of growth inhibitory genes or by activating the expression of pro-growth genes. to therapeutically control cardiac remodeling by directly inhibiting chromatin-modifying enzymes. In this regard, synthetic and naturally occurring small molecule inhibitors of HATs have been described $(64,65)$. Several classes of enzymatic inhibitors of HDACs, including hydroxamic acids, short chain fatty acids, benzamides, cyclic tetrapeptides, and bicyclic depsipeptides, have also been identified (66).

The effect of HAT inhibitors on cardiac remodeling remains unknown. However, given the ability of the p300 HAT to stimulate dilated cardiomyopathy in mice (67), this class of compounds may prove beneficial in the context of heart failure. Surprisingly, HDAC inhibitors have been found to potently repress agonist-dependent cardiac hypertrophy in a manner that correlates with increased histone acetylation $(68,69)$. This finding was paradoxical, since class II HDACs block cardiac hypertrophy, and HDAC inhibitors would logically be predicted to neutralize this repressive function.

How might the paradoxical effects of class II HDACs and HDAC inhibitors on cardiac growth be explained? The demonstration that class II HDAC catalytic activity is not required to repress the hypertrophic program suggests that these HDACs act through a mechanism independent of HDAC activity to repress hypertrophy (26). The antihypertrophic action of class II HDACs likely lies in their capacity to prohibit binding of HATs to MEF2 or other transcription factors and to repress transcription via HDAC-independent mechanisms (13).

Based on the results with HDAC inhibitors, one or more HDACs may play a positive or permissive role in the control of cardiac hypertrophy. Given the antihypertrophic function of class II HDACs, a class I HDAC(s) likely fulfills this role. Of note, the HDAC inhibitors used in studies of cardiac hypertrophy do not antagonize NAD-dependent class III HDACs. A testable prediction of this model is that overexpression of a class I HDAC in the heart will cause pathological hypertrophy.

The mechanism for HDAC inhibitor-mediated repression of cardiac hypertrophy remains unknown. We envision at least 3 possibilities (Figure 8). (a) HDACs may be required to block expression of genes that encode repressors of cardiac growth. (b) HDACs may stimulate expression of a pro-cardiac growth gene(s). In this regard, although HDACs are typically associated with gene repression, there is an increasing number of examples in which HDACs have been linked to gene induction (70). (c) HDACs may directly or indirectly modulate prohypertrophic signal transduction cascades. Indeed, several nonhistone targets for HATs/HDACs have been identified, including factors implicated in cardiac remodeling, such as tubulin and the GATA transcription factor $(67,71)$.

\section{Potential for HDAC inhibitors in heart failure therapy}

The fortuitous discovery that HDAC inhibitors repress cardiac hypertrophy and normalize cardiac gene expression in the face of stress may ultimately affect the treatment of heart failure in humans. Importantly, HDAC inhibition in vitro and in vivo results in downregulation $\beta$-MHC expression, with a concomitant increase in the levels of $\alpha$-MHC $(68,72)$. Thus, we predict that HDAC inhibitors will not only antagonize deleterious cardiac growth, but will also increase myofibrillar ATPase activity and improve contractility in the failing heart.

Advancement of an HDAC inhibitor into the clinic for the treatment of heart failure awaits rigorous preclinical testing in animal models of pathological cardiac remodeling. Nonetheless, enthusiasm for this novel therapeutic approach is buoyed by successes with HDAC inhibitors in other disease models as well as in humans. Indeed, at least 9 independent approaches for HDAC inhibition are currently being tested in human clinical trials for cancer, and therapeutic benefit and tolerability have been observed (66).

\section{Looking to the future}

The primary focus of this review has been on pathways and interventions that affect cardiomyocyte hypertrophy as an endpoint. While cardiac hypertrophy is clearly an important prognostic indicator of poor clinical outcome (2-5), it is but one component of the remodeling process, and deleterious remodeling can occur in the absence of cardiac cell growth. A common feature of remodeling, regardless of etiology, is fetal cardiac gene induction, which is thought to contribute to cardiac demise through the dysregulation of genes encoding proteins that control cardiac contractility (e.g., MHC). The types of transcriptional therapies proposed here would be designed to block not hypertrophy per se, but rather the pathological gene program that underlies the remodeling process.

Despite the success of lipid-lowering drugs for the prevention of coronary artery disease and myocardial infarction, and several decades of effort toward modifying cardiac function through traditional drug targets, heart failure remains the most common cause of disease-related death in the Western world, and 
all projections for the future point to a continued increase in its prevalence. Thus, major advances toward the reduction of heart failure incidence will require new therapeutic approaches. We propose that pathological cardiac remodeling in the settings of hypertrophy and heart failure can be viewed, at least in part, as a transcriptional disorder and that normalization of the gene expression pattern of the cardiac myocyte through transcriptional therapies represents a promising and largely unexploited approach for cardiac therapy. Recent advances in understanding stress-response pathways responsible for heart failure and mechanisms of cardiac gene expression, combined with new technologies for high throughput screening for novel small molecular modifiers of cellular functions, offer promising and untapped opportunities for success in failure.

1. Linseman, J.V., and Bristow, M.R. 2003. Drug therapy and heart failure prevention. Circulation. 107:1234-1236.

2. Vakili, B.A., Okin, P.M., and Devereux, R.B. 2001. Prognostic implications of left ventricular hypertrophy. Am. Heart J. 141:334-341.

3. Okin, P.M., et al.; for the LIFE Study Investigators. 2004. Regression of electrocardiographic left ventricular hypertrophy during antihypertensive treatment and the prediction of major cardiovascular events. JAMA. 292:2343-2349.

4. Devereux, R.B., et al. 2004. Prognostic significance of left ventricular mass change during treatment of hypertension. JAMA. 292:2350-2356.

5. Gardin, J.M., and Lauer, M.S. 2004. Left ventricular hypertrophy: the next treatable, silent killer? JAMA. 292:2396-2398.

6. Olson, E.N., and Schneider, M.D. 2003. Sizing up the heart: development redux in disease. Genes Dev. 17:1937-1956.

7. Chien, K.R. 1999. Stress pathways and heart failure. Cell. 98:555-558.

8. Marks, A.R. 2003. A guide for the perplexed: towards an understanding of the molecular basis of heart failure. Circulation. 107:1456-1459.

9. Molkentin, J.D., and Dorn, I.G., 2nd. 2001. Cytoplasmic signaling pathways that regulate cardiac hypertrophy. Annu. Rev. Physiol. 63:391-426.

10. Abraham, W.T., et al. 2002. Coordinate changes in Myosin heavy chain isoform gene expression are selectively associated with alterations in dilated cardiomyopathy phenotype. Mol. Med. 8:750-760.

11. Lowes, B.D., et al. 2002. Myocardial gene expression in dilated cardiomyopathy treated with betablocking agents. N. Engl. J. Med. 346:1357-1365.

12. Blaxall, B.C., Tschannen-Moran, B.M., Milano, C.A., and Koch, W.J. 2003. Differential gene expression and genomic patient stratification following left ventricular assist device support. J. Am. Coll. Cardiol. 41:1096-1106.

13. McKinsey, T.A., and Olson, E.N. 2004. Cardiac histone acetylation--therapeutic opportunities abound. Trends Genet. 20:206-213.

14. Braunwald, E., and Bristow, M.R. 2000. Congestive heart failure: fifty years of progress. Circulation. 102(Suppl. 4):IV14-IV23.

15. Herron, T.J., and McDonald, K.S. 2002. Small amounts of alpha-myosin heavy chain isoform expression significantly increase power output of rat cardiac myocyte fragments. Circ. Res. 90:1150-1152.

16. Nakao, K., Minobe, W., Roden, R., Bristow, M.R., and Leinwand, L.A. 1997. Myosin heavy chain gene expression in human heart failure. J. Clin. Invest. 100:2362-2370.

17. Miyata, S., Minobe, W., Bristow, M.R., and Leinwand, L.A. 2000. Myosin heavy chain isoform expression in the failing and nonfailing human heart. Circ. Res. 86:386-390.

18. Niimura, H., et al. 2002. Sarcomere protein gene mutations in hypertrophic cardiomyopathy of the elderly. Circulation. 105:446-451.

19. Ashrafian, H., Redwood, C., Blair, E., and Watkins, H. 2003. Hypertrophic cardiomyopathy: a paradigm for myocardial energy depletion. Trends Genet. 19:263-268.

20. Sano, M., et al. 2002. Activation and function of cyclin T-Cdk9 (positive transcription elongation factor-b) in cardiac muscle-cell hypertrophy. Nat. Med. 8:1310-1317.

21. Sugden, P.H., and Clerk, A. 1998. "Stress-responsive" mitogen-activated protein kinases (c-Jun $\mathrm{N}$-terminal kinases and p38 mitogen-activated protein kinases) in the myocardium. Circ. Res. 83:345-352.

22. Frey, N., and Olson, E.N. 2003. Cardiac hypertrophy: the good, the bad, and the ugly Annu. Rev. Physiol. 65:45-79.

23. Rockman, H.A., Koch, W.J., and Lefkowitz, R.J. 2002. Seven-transmembrane-spanning receptors and heart function. Nature. 415:206-212.

24. Fischle, W., Wang, Y., and Allis, C.D. 2003. Histone and chromatin cross-talk. Curr. Opin. Cell Biol. 15:172-183.

25. Verdin, E., Dequiedt, F., and Kasler, H.G. 2003. Class II histone deacetylases: versatile regulators. Trends Genet. 19:286-293.

26. Zhang, C.L., et al. 2002. Class II histone deacetylases act as signal-responsive repressors of cardiac hypertrophy. Cell. 110:479-488.

27. Bush, E., et al. 2004. A small molecular activator of cardiac hypertrophy uncovered in a chemical screen for modifiers of the calcineurin signaling pathway. Proc. Natl. Acad. Sci. U. S. A. 101:2870-2875.

28. Vega, R.B., et al. 2004. Protein kinases C and D mediate agonist-dependent cardiac hypertrophy through nuclear export of histone deacetylase 5 . Mol. Cell. Biol. 24:8374-8385.

29. Chang, S., et al. 2004. Histone deacetylases 5 and 9 govern responsiveness of the heart to a subset of stress signals and play redundant roles in heart. Mol. Cell. Biol. 24:8467-8476.

30. McKinsey, T.A., Zhang, C.L., and Olson, E.N. 2002. MEF2: a calcium-dependent regulator of cell division, differentiation and death. Trends Biochem. Sci. 27:40-47.

31. Harrison, B.C., et al. 2004. The CRM1 nuclear export receptor controls of pathological cardiac gene expression. Mol. Cell. Biol. 24:10636-10649.

32. Youn, H.D., Grozinger, C.M., and Liu, J.O. 2000. Calcium regulates transcriptional repression of myocyte enhancer factor 2 by histone deacetylase 4 . J. Biol. Chem. 275:22563-22567.

33. Han, A., et al. 2003. Sequence-specific recruitment of transcriptional co-repressor Cabin 1 by myocyte enhancer factor-2. Nature. 422:730-734.

34. McKinsey, T.A., Zhang, C.L., Lu, J., and Olson, E.N. 2000. Signal-dependent nuclear export of a histone deacetylase regulates muscle differentia- tion. Nature. 408:106-111.

35. McKinsey, T.A., Zhang, C.L., and Olson, E.N. 2000. Activation of the myocyte enhancer factor- 2 transcription factor by calcium/calmodulin-dependent protein kinase-stimulated binding of 14-3-3 to histone deacetylase 5. Proc. Natl. Acad. Sci. U. S. A. 97:14400-14405.

36. Grozinger, C.M., and Schreiber, S.L. 2000. Regulation of histone deacetylase 4 and 5 and transcriptional activity by 14-3-3-dependent cellular localization. Proc. Natl. Acad. Sci. U. S. A. 97:7835-7840.

37. McKinsey, T.A., Zhang, C.L., and Olson, E.N. 2001. Identification of a signal-responsive nuclear export sequence in class II histone deacetylases. Mol. Cell. Biol. 21:6312-6321.

38. Wang, A.H., and Yang, X.J. 2001. Histone deacetylase 4 possesses intrinsic nuclear import and export signals. Mol. Cell. Biol. 21:5992-6005.

39. Das, D.K. 2003. Protein kinase C isozymes signaling in the heart. J. Mol. Cell. Cardiol. 35:887-889.

40. Force, T., Kuida, K., Namchuk, M., Parang, K., and Kyriakis, J.M. 2004. Inhibitors of protein kinase signaling pathways: emerging therapies for cardiovascular disease. Circulation. 109:1196-1205.

41. Gschwendt, M., et al. 1996. Inhibition of protein kinase $\mathrm{C} \mathrm{mu}$ by various inhibitors. Differentiation from protein kinase c isoenzymes. FEBS Lett. 392:77-80.

42. Haworth, R.S., et al. 2004. Protein kinase D is a novel mediator of cardiac troponin I phosphorylation and regulates myofilament function. Circ Res. 95:1091-1099.

43. Van Lint, J., et al. 2002. Protein kinase D: an intracellular traffic regulator on the move. Trends Cell Biol. 12:193-200.

44. Han, J., and Molkentin, J.D. 2000. Regulation of MEF2 by p38 MAPK and its implication in cardiomyocyte biology. Trends Cardiovasc. Med. 10:19-22.

45. Youn, H.D., Chatila, T.A., and Liu, J.O. 2000. Integration of calcineurin and MEF2 signals by the coactivator p300 during T-cell apoptosis. EMBO J. 19:4323-4231.

46. Naya, F.J., et al. 2002. Mitochondrial deficiency and cardiac sudden death in mice lacking the MEF2A transcription factor. Nat. Med. 8:1303-1309.

47. Wang, L., Fan, C., Topol, S.E., Topol, E.J., and Wang, Q. 2003. Mutation of MEF2A in an inherited disorder with features of coronary artery disease. Science. 302:1578-1581.

48. Czubryt, M.P., McAnally, J., Fishman, G.I., and Olson, E.N. 2003. Regulation of peroxisome proliferator-activated receptor gamma coactivator 1 alpha (PGC-1 alpha) and mitochondrial function by MEF2 and HDAC5. Proc. Natl. Acad. Sci. U. S. A. 100:1711-1716.

49. Sano, M., et al. 2004. Activation of cardiac Cdk9 represses PGC-1 and confers a predisposition to heart failure. EMBO J. 23:3559-3569. 
50. Molkentin, J.D., et al. 1998. A calcineurin-dependent transcriptional pathway for cardiac hypertrophy. Cell. 93:215-228.

51. Leinwand, L.A. 2001. Calcineurin inhibition and cardiac hypertrophy: a matter of balance. Proc. Natl. Acad. Sci. U. S. A. 98:2947-2949.

52. Antos, C.L., et al. 2002. Activated glycogen synthase kinase-3 beta suppresses cardiac hypertrophy in vivo. Proc. Natl. Acad. Sci. U. S. A. 99:907-912.

53. Haq, S., et al. 2000. Glycogen synthase kinase-3beta is a negative regulator of cardiomyocyte hypertrophy. J. Cell Biol. 151:117-130.

54. Morisco, C., et al. 2000. The Akt-glycogen synthase kinase 3 beta pathway regulates transcription of atrial natriuretic factor induced by beta-adrenergic receptor stimulation in cardiac myocytes. J. Biol. Chem. 275:14466-14475.

55. Frey, N., Katus, H.A., Olson, E.N., and Hill, J.A. 2004. Hypertrophy of the heart: a new therapeutic target? Circulation. 109:1580-1589.

56. Vega, R.B, Bassel-Duby, R., and Olson, E.N. 2003. Control of cardiac growth and function by calcineurin signaling. J. Biol. Chem. 278:36981-36984.

57. Yang, J., et al. 2000. Independent signals control expression of the calcineurin inhibitory proteins MCIP1 and MCIP2 in striated muscles. Circ. Res. 87:E61-E68.

58. Vega, R.B., et al. 2003. Dual roles of modulatory calcineurin-interacting protein 1 in cardiac hypertrophy. Proc. Natl. Acad. Sci. U. S. A. 100:669-674.

59. Rothermel, B.A., et al. 2001. Myocyte-enriched calcineurin-interacting protein, MCIP1, inhibits cardiac hypertrophy in vivo. Proc. Natl. Acad. Sci. U. S. A 98:3328-3333.

60. Hill, J.A., et al. 2002. Targeted inhibition of calcineurin in pressure-overload cardiac hypertrophy. Preservation of systolic function. J. Biol. Chem. 277:10251-10255.

61. van Rooij, E., et al. 2004. MCIP1 overexpression suppresses left ventricular remodeling and sustains cardiac function after myocardial infarction. Circ. Res. 94:e18-e26.

62. Nebigil, C.G., et al. 2003. Overexpression of the serotonin 5-HT2B receptor in heart leads to abnormal mitochondrial function and cardiac hypertrophy. Circulation. 107:3223-3229.

63. Nebigil, C.G., et al. 2001. Ablation of serotonin 5 -HT(2B) receptors in mice leads to abnormal cardiac structure and function. Circulation. 103:2973-2979.

64. Lau, O.D., et al. 2000. HATs off: selective synthetic inhibitors of the histone acetyltransferases p300 and PCAF. Mol. Cell. 5:589-595.

65. Balasubramanyam, K., Swaminathan, V., Ranganathan, A., and Kundu, T.K. 2003. Small molecule modulators of histone acetyltransferase p300. J. Biol. Chem. 278:19134-19140
66. Piekarz, R., and Bates, S. 2004. A review of depsipeptide and other histone deacetylase inhibitors in clinical trials. Curr. Pharm. Des. 10:2289-2298.

67. Yanazume, T., et al. 2003. Cardiac p300 is involved in myocyte growth with decompensated heart failure. Mol. Cell. Biol. 23:3593-3606.

68. Antos, C.L., et al. 2003. Dose-dependent blockade to cardiomyocyte hypertrophy by histone deacetylase inhibitors. J. Biol. Chem. 278:28930-28937.

69. Kook, H., et al. 2003. Cardiac hypertrophy and histone deacetylase-dependent transcriptional repression mediated by the atypical homeodomain protein Hop. J. Clin. Invest. 112:863-871. doi:10.1172/JCI200319137

70. Chang, H.M., et al. 2004. Induction of interferonstimulated gene expression and antiviral responses require protein deacetylase activity. Proc. Natl. Acad. Sci. U. S. A. 101:9578-9583.

71. Westermann, S., and Weber, K. 2003. Post-translational modifications regulate microtubule function. Nat. Rev. Mol. Cell Biol. 4:938-947.

72. Davis, F.J., Pillai, J.B., Gupta, M., and Gupta, M.P. 2005. Concurrent opposite effects of an inhibitor of histone deacetylases, Trichostatin-A, on the expression of cardiac \{alpha\}-myosin heavy chain and tubulins: implication for gain in cardiac muscle contractility. Am. J. Physiol. Heart Circ. Physiol. 288:H1477-H1490. 